\title{
Flow-induced dynamic surface tension effects at nanoscale
}

Article

Accepted Version

Lukyanov, A. V. (2010) Flow-induced dynamic surface tension effects at nanoscale. Langmuir, 26 (9). pp. 6367-6373. ISSN 0743-7463 doi: https://doi.org/10.1021/la9040453 Available at https://centaur.reading.ac.uk/17197/

It is advisable to refer to the publisher's version if you intend to cite from the work. See Guidance on citing.

Published version at: http://pubs.acs.org/doi/abs/10.1021/la9040453

To link to this article DOI: http://dx.doi.org/10.1021/la9040453

Publisher: American Chemical Society

All outputs in CentAUR are protected by Intellectual Property Rights law, including copyright law. Copyright and IPR is retained by the creators or other copyright holders. Terms and conditions for use of this material are defined in the End User Agreement.

\section{www.reading.ac.uk/centaur}

\section{CentAUR}

Central Archive at the University of Reading

Reading's research outputs online 


\title{
Flow-Induced Dynamic Surface Tension Effects at Nanoscale
}

\author{
A. V. Lukyanov \\ Department of Mathematics, University of Reading, Reading RG6 6AX, United Kingdom
}

Received October 25, 2009. Revised Manuscript Received January 26, 2010

\begin{abstract}
The aim of this study is to investigate flow-induced dynamic surface tension effects, similar to the well-known Marangoni phenomena, but solely generated by the nanoscale topography of the substrates. The flow-induced surface tension effects are examined on the basis of a sharp interface theory. It is demonstrated how nanoscale objects placed at the boundary of the flow domain result in the generation of substantial surface forces acting on the bulk flow.
\end{abstract}

\section{Introduction}

The distinctive feature of fluid motion at nanoscale is its strong coupling with the dynamic processes in the interfacial layers formed at the boundaries between the phases. ${ }^{1,2}$ For example, we know from recent experiments that slippage of liquids at solid substrates results in enhanced liquid transport through nanoscale capillary channels, up to 45-400 times higher in comparison to the theoretical predictions based on the no-slip boundary condition. ${ }^{3,4}$ The slippage of liquids is only one manifestation of interfacial dynamic properties. Another effect associated with the formation of interfacial layers and widely exploited to control nanoflows is surface tension. ${ }^{5-9}$

If a moving contact line is present, the dynamic surface tension effects manifest themselves in the dependence of the contact angle $\theta_{\mathrm{d}}\left(u_{\mathrm{c}}\right)$ formed between the moving free surface and the solid substrate on the velocity of the triple-phase contact line, $u_{\mathrm{c}}{ }^{6,7,9}$ This can be illustrated by the Young equation, $\cos \theta_{\mathrm{d}} \sigma_{\mathrm{GL}}=-\sigma_{\mathrm{LS}}$ $+\sigma_{\mathrm{GS}}$, where $\sigma_{\mathrm{GL}}, \sigma_{\mathrm{LS}}$, and $\sigma_{\mathrm{GS}}$ are the surface tensions of gas-liquid, liquid-solid, and gas-solid interfaces, evaluated at the contact line. The dependence $\theta_{\mathrm{d}}\left(u_{\mathrm{c}}\right)$ implies that at least some of the surface tensions are not equal to their equilibrium values when the contact line is at rest. The velocity dependence of the dynamic contact angle is only one part of its general dependence; apart from the substrate velocity, the contact angle is a function of the entire flow field at the contact line region. This effect is known as the nonlocality of dynamic contact angle or, in macroscopic context, as the hydrodynamic assist of dynamic wetting, which has been used for decades in the coating industries. ${ }^{10,11}$

Studies of the effects of dynamic wetting on smooth flat surfaces (in particular the effect of nonlocality) have revealed several characteristic features of the interfacial phase dynamics. Slippage of liquids and the dynamic surface tensions are closely interrelated; the $\sigma_{\mathrm{GL}}$ and $\sigma_{\mathrm{LS}}$ surface tensions at the contact line deviate from the equilibrium values

(1) Zhao, B.; Moore, J. S.; Beebe, D. J. Anal. Chem. 2002, 74, 4259-4268.

(2) Squires, T. M.; Quake, S. R. Rev. Mod. Phvs. 2005, 77, 977-1026

(3) Whitby, M.; Cagnon, L.; Thanou, M.; Quirke, N. Nano Lett. 2008, 8, 26322637.

(4) Thomas, J. A.; McGaughey, A. J. H. Nano Lett. 2008, 8, 2788-2793.

(5) Darhuber, A. A.; Troian, S. M. Annu. Rev. Fluid Mech. 2005, 37, 425-455.

(6) Blake, T. D. J. Colloid Interface Sci. 2006, 299, 1-13.

(7) Shikhmurzaev, Y. D. (2007) Capillary Flows with Forming Interfaces; Taylor \& Francis.

(8) Bonn, D.; Eggers, J.; Indekeu, J.; Meunier, J.; Rolley, E. Rev. Mod. Phys. 2009, 81, 739-805.

(9) Ralston, J.; Popescu, M.; Sedev, R. Annu. Rev. Mater. Res. 2008, 38, 23-43.

(10) Blake, T. D.; Clarke, A.; Rushak, K. J. AIChE J. 1994, 40, 229-242.

(11) Yamamura, M. Colloids Surf. A 2007, 311, 55-60. and equilibrate over the distance, away from the contact line region, defined by the characteristic diffusion time in the interfacial layer, that is, by the characteristic time of the formation of the interfacial layers. ${ }^{7,10-17}$ This scenario has been further supported by the evidence from independent studies of viscous flows over the surfaces with variable wettability, where the changes of the liquid-solid interfacial energy $\sigma_{\mathrm{LS}}(x)$ have been achieved by chemical patterning of the substrate. ${ }^{18,19}$

In this paper, we investigate another effect, which is associated with forming interfaces and especially relevant to nanofluidic flows, of coupling of the surface topography and the flow-induced surface tensions. The question is, what if we change the flat geometry of the solid surface by placing a tiny, nanoscale obstacle on the surface? The effect of a particle arrested on a substrate is well-studied for macroscopic incompressible flows. ${ }^{20,21}$ However, if the obstacle is, approximately, the size of the interfacial layer, where the incompressibility condition is relaxed to account for surface tension, then one would expect to observe completely new effects, since the presence of an obstacle may disturb the surface phase density equilibrium state (by compressing the liquid in the interfacial layer, for example) and cause variations in the surface tension, similar to the well-known Marangoni phenomenon, though in this case solely induced by the surface topography. In this connection, we note that the size of the interfacial layer, $h$, is on the order of a few nanometers, $h \simeq 1-4 \mathrm{~nm}$, for simple fluids ${ }^{22}$ so that already nanoscale objects should be able to affect the equilibrium state of the surface phase at the substrates.

From the experimental point of view, several observations, especially using the fluorescence recovery technique and nanoparticles seeded on the substrate in a controlled manner, ${ }^{23-25}$

(12) Blake, T. D.; Bracke, M; Shikhmurzaev, Y. D. Phvs. Fluids 1999, 11, 1995 2007.

(13) Wilson, M. C. T.; Summers, J. L.; Shikhmurzaev, Y. D.; Clarke, A; Blake, T. D. Phvs. Rev. E 2006, 73, 041606

(14) Bayer, I. S.; Megaridis, C. M. J. Fluid Mech. 2006, 558, 415-449.

(15) Clarke, A.; Stattersfield, E. Phvs. Fluids 2006, 18, 048106.

(16) Lukyanov, A.; Shikhmurzaev, Y. D. Phvs. Lett. A 2006, 358, 426-430.

(17) Lukyanov, A.; Shikhmurzaev, Y. D. Phvs. Rev. E 2007, 75, 051604.

(18) Priezjev, N. V.; Darhuber, A. A.; Troian, S. M. Phvs. Rev. E 2005, 71, 041608 .

(19) Sprittles, J. E.; Shikhmurzaev, Y. D. Phvs. Rev. E 2007, 76, 021602.

(20) Pozrikidis, C.; Thoroddsen, S. T. Phvs. Fluids A 1991, 3, 2546-2558.

(21) Blyth, M. G.; Pozrikidis, C. Phvs. Fluids 2006, 18, 052104.

(22) Derjaguin, B. V.; Churaev, N. V.; Muller, V. M. Surface Forces; Kitchener,

J. A., Ed.; Consultants Bureau/Plenum Publishing Co.: New York, 1987.

(23) Zhu, Y.; Granick, S. Phvs. Rev. Lett. 2002, 88, 106102.

(24) Guriyanova, S.; Semin, B.; Rodrigues, T. S.; Butt, H. J.; Bonaccurso, E. (2010) Microfluidics Nanofluidics, In press.

(25) Schmatko, T.; Hervet, H.; Leger, L. Langmuir 2006, 22, 6843-6850. 


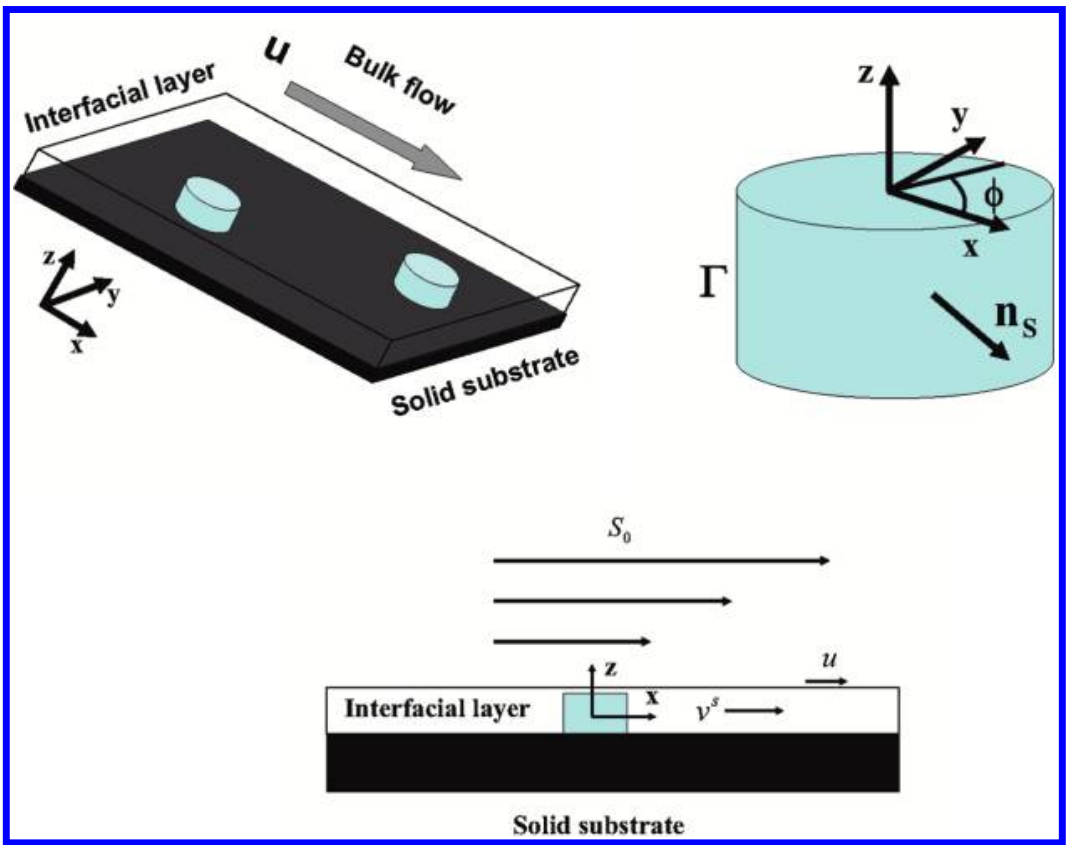

Figure 1. Definition sketch for the problem.

have indicated that nanoscale topography on the length scale of interfacial layer has indeed a strong effect on the flow conditions at the substrates, in particular, on the amount of the observed slip. The minimum height of the surface irregularities, when the effect of their presence can still be observed, was on the length scale of the interfacial layer for simple fluids used in the experiments, such as hexadecane and tetradecane, for example. This, in particular, implies that a substrate can be regarded as a flat one in the context of nanoflows and the current study, if variations of the surface profile are below the threshold of the order of a few nanometers. One needs to note here, however, that on the experimental side the whole picture is still not crystal clear at the moment, since, for instance, experimental results obtained using an atomic force microscope ${ }^{24}$ seem to contradict qualitatively experimental observations obtained using the fluorescence recovery method. ${ }^{25}$

\section{Theoretical Model}

The analysis of the topography-induced surface tension problem is based on the sharp interface formation theory, ${ }^{7}$ given the length scale of the interfacial region of a few nanometers, ${ }^{22}$ coupled with the effect of surface slip. ${ }^{26,27}$ This approach, first developed using methods of nonequilibrium thermodynamics, ${ }^{28}$ is based on the standard set of the Navier-Stokes equations, taken in the context of nanoflows with negligible inertia, for the flow velocity $\mathbf{u}$ and pressure $p$ in the bulk of an incompressible and, for simplicity, Newtonian liquid with viscosity $\mu$ and density $\rho$

$$
\nabla \cdot \mathbf{u}=0 \quad \nabla p=\mu \nabla^{2} \mathbf{u}
$$

and an extended set of boundary conditions, ${ }^{7}$ at the solid substrate, at $z=0$, which describes a coupling between the bulk phase, the surface phase in the interfacial layer, and the solid

(26) Bocquet., L.; Barrat, J. L. Phvs. Rev. E 1994, 49, 3079-3092.

(27) Barrat, J. L.; Bocquet., L. Phvs. Rev. Lett. 1999, 82, 4671-4674.

(28) Bedeaux, D.; Albano, A. M.; Mazur, P. Phvsica A 1975, 82, 438-462. substrate (see Figure 1). In the sharp interface limit, the thickness of the interfacial layer is zero and the surface phase is solely characterized by two-dimensional distributions of the surface velocity $\mathbf{v}^{\mathbf{s}}$ and surface density $\rho^{s}$, which are integrated values over the interfacial layer. The solid substrate is impermeable, so

$$
\mathbf{v}^{\mathrm{s}} \cdot \mathbf{n}=0
$$

where $\mathbf{n}$ is the normal vector at the substrate pointing into the liquid. The second boundary condition is the tangential stress balance equation in the interfacial layer, which is, in fact, a modified Navier condition, ${ }^{7}$ with the coefficient of surface slip $\beta_{\mathrm{s}}{ }^{26,27}$ This condition also takes into account the Marangoni effect where the flow is driven by the surface tension gradient $\nabla \sigma$ and thus links the effects of slip and surface tension $^{7,19}$

$$
\mu \mathbf{n} \cdot\left[\nabla \mathbf{u}+(\nabla \mathbf{u})^{*}\right] \cdot(\mathbf{I}-\mathbf{n n})+\nabla \sigma=\beta_{\mathrm{s}} \mathbf{u} \cdot(\mathbf{I}-\mathbf{n n})
$$

Here, $\mathbf{I}$ is the metric tensor; the tensor $(\mathbf{I}-\mathbf{n n})$ singles out the tangential projection of a vector; an asterisk marking a second-rank tensor indicates its transposition. Note that we have neglected the effect of apparent slip, so the tangential component of the velocity $\mathbf{u}$ on the liquid-facing side of the interface, as is shown in Figure 1, is simply equal to the surface phase velocity

$$
\mathbf{v}^{\mathrm{s}} \cdot(\mathbf{I}-\mathbf{n n})=\mathbf{u} \cdot(\mathbf{I}-\mathbf{n n})
$$

Such simplification is possible if the observed slip length satisfies certain conditions. We note, first, that the notion of apparent slip appears only in the macroscopic modeling of interfaces and represents the fact that the thickness of the interfacial layer is below the spatial resolution of the NavierStokes equations. As a result, first of all, the macroscopic boundary conditions for the Navier-Stokes equations are to be specified on the liquid-facing side of the interfacial layer. Second, there should be a difference between the tangential components of 
the hydrodynamic velocity on the liquid-facing and solid-facing sides of the interface, which can be regarded as the apparent slip with the corresponding coefficient of sliding friction $\beta_{a}$ or apparent slip length $\lambda_{a}=\mu / \beta_{a}{ }^{7}$ The surface slip, at variance to the apparent slip, is effectively the difference between the tangential components of the substrate velocity and the hydrodynamic velocity on the solid-facing side of the interfacial layer. As has been shown previously, ${ }^{29}$ those two slip effects are additive. An upper bound of the apparent slip length can be found using estimates obtained from experiments on dynamic wetting, ${ }^{30}$ that is, $\beta_{a} \simeq \mu_{a} / h$, where $h$ is the thickness of the interfacial layer, which is on the order of a few nanometers, ${ }^{22}$ and $\mu_{a}$ is the average viscosity in the interfacial layer. So, it appears that the apparent slip length is roughly $\lambda_{a} \simeq h$, if the viscosity in the interfacial layer $\mu_{a} \simeq \mu$ is not much different from the bulk viscosity. This implies that the apparent slip can be neglected if $\lambda \gg h$, which will be further assumed. Note, however, that the viscosity at the boundary may be several times lower than the values in the bulk for some fluids, especially with long-chain molecules, ${ }^{22}$ though this factor is not significant for our current study.

The set of boundary conditions is completed by two equations describing the mass exchange between the bulk and the surface

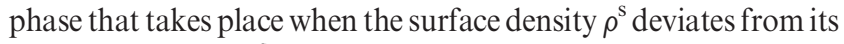
equilibrium value $\rho_{\mathrm{e}}^{\mathrm{s}}$

$$
\begin{gathered}
\rho \mathbf{u} \cdot \mathbf{n}=\left(\rho^{\mathrm{s}}-\rho_{\mathrm{e}}^{\mathrm{s}}\right) \tau^{-1} \\
\frac{\partial \rho^{\mathrm{s}}}{\partial t}+\nabla \cdot\left(\rho^{\mathrm{s}} \mathbf{v}^{\mathrm{s}}\right)=-\left(\rho^{\mathrm{s}}-\rho_{\mathrm{e}}^{\mathrm{s}}\right) \tau^{-1}
\end{gathered}
$$

and an equation of state relating the surface tension and the surface density, which is taken for simplicity in a linear form

$$
\sigma=\gamma\left(\rho_{0}^{\mathrm{s}}-\rho^{\mathrm{s}}\right)
$$

Here, parameter $\tau$ is the surface density relaxation time, $\rho_{0}^{\mathrm{s}}$ is the characteristic surface density when the surface tension is zero, and $\gamma$ is a phenomenological material constant.

We consider a steady-state solution to eqs $1-7$, assuming that the flow is driven by a plane-parallel constant shear $\mathbf{S}_{0}$ in the far field, which is directed, without loss of generality, along the $x$-axis (see Figure 1). To account for the presence of a particle on the substrate, one needs to specify how the particle interacts with the surface phase flow. In an ideal situation, such information can be obtained from a microscopic consideration, such as molecular dynamics simulations. In the context of this macroscopic study, we will leave the microscopic consideration for future work and postulate these conditions by making reasonable assumptions. As we will see further, effectively one needs to specify only one boundary condition, which is the flux of the surface phase at the boundary of obstacles. Once the flux is specified, the problem is fully and uniquely defined, at least in the asymptotic limit, which we are going to use.

In a simplified two-dimensional case, in the $(x, z)$ plane, of a one-dimensional nanothread lying on the solid substrate normal to the flow (see Figure 2 for illustration), the problem in eqs $1-7$ has been analyzed previously in the context of the effect of slip. ${ }^{29}$ We will recapitulate briefly the main results of the previous study, ${ }^{29}$ which will be later used for comparison. It has been

(29) Lukyanov, A. Phvs. Lett. A 2009, 373, 1967-1971. 202 .

(30) Blake, T. D.; Shikhmurzaev, Y. D. J. Colloid Interface Sci. 2002, 253, 196-

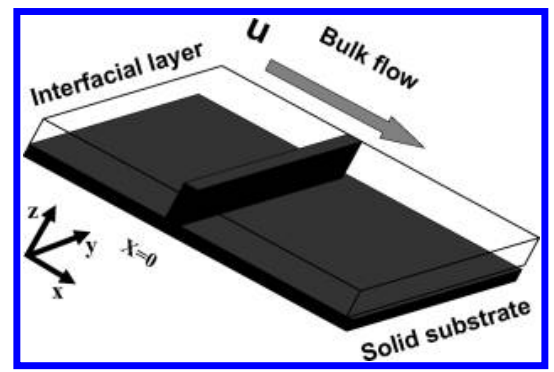

Figure 2. Definition sketch for the simplified two-dimensional problem with one nanothread on the surface at $x=0$.

shown that, if we use $L=\lambda=\mu / \beta_{s}, U_{0}=L S_{0}, p_{0}=\mu U_{0} / L$, and $\rho_{\mathrm{e}}{ }^{\mathrm{s}}$ as scales for velocity, length, pressure, and surface density, the normalization we will use further in the paper, and $\lambda$ is the characteristic slip length, then the problem has three essential nondimensional parameters $\epsilon=U_{0} \tau / \lambda, \mathrm{Ca}=\mu U_{0} / \sigma_{0}$, and $Q=$ $\rho_{\mathrm{e}}^{\mathrm{s}} / \rho \lambda$; parameter $\sigma_{0}=\gamma \rho_{\mathrm{e}}^{\mathrm{s}}$ is the characteristic surface tension; $\gamma$ is inversely proportional to the fluid's compressibility and is, roughly, the square of the speed of sound; $\rho_{\mathrm{e}}^{\mathrm{s}} \sim \rho_{0}^{\mathrm{s}} \sim \rho h$, where $h$ is the interfacial thickness. Parameter $\epsilon$ is the ratio of the characteristic relaxation length $U_{0} \tau$ to the characteristic slip length $\lambda, \mathrm{Ca}$ is the capillary number, parameter $Q \simeq h / \lambda$ characterizes the mass flux into/out of the liquid-solid interface. It is common that $\mathrm{Ca} \ll 1, Q \ll 1$, and $\epsilon \ll 1$, while the ratio $\mathrm{Ca} / \epsilon=\xi_{0}^{2} \sim O(1)$. For example, using $S_{0} \sim 10^{4} \mathrm{~s}^{-1}, \lambda \sim 100 \mathrm{~nm},{ }^{31} \sigma_{0} \sim 10^{-1} \mathrm{~N} / \mathrm{m}\left(\rho_{\mathrm{e}}^{\mathrm{s}} \sim\right.$ $\left.10^{-6} \mathrm{~kg} / \mathrm{m}^{2}, \gamma \sim 10^{5} \mathrm{~m}^{2} / \mathrm{s}^{2}\right), \mu \sim 10^{-1} \mathrm{~Pa} \mathrm{~s}$, and $\tau \sim 10^{-8} \mathrm{~s}$ as estimates of the characteristic shear rate, slip length, surface tension, viscosity, and relaxation time, $U_{0} \sim 10^{-3} \mathrm{~m} / \mathrm{s}$ and $\mathrm{Ca} \sim$ $10^{-3}, \epsilon \sim 10^{-2}$, and $Q \sim 3 \times 10^{-2}$. Here, parameter $\tau$ is taken, according to the estimates obtained from experiments on dynamic wetting, ${ }^{30}$ to scale for simple fluids as $\tau=10^{-8}(\mu / 1 \mathrm{mPa} \mathrm{s}) \mathrm{s}$. Then, in the asymptotic limit $\epsilon \rightarrow 0, \mathrm{Ca} / \epsilon=\xi_{0}^{2} \sim \mathrm{O}(1)$, the problem in eqs $1-7$ is found to be equivalent to solving a secondorder ordinary differential equation

$$
\begin{gathered}
\frac{\mathrm{d}^{2} \rho_{1}{ }^{\mathrm{s}}}{\mathrm{d} x^{2}}-\xi_{0}{ }^{2} \rho_{1}{ }^{\mathrm{s}}=0, \quad x \in \mathbb{R} \\
\rho^{\mathrm{s}}=1+\epsilon \rho_{1}{ }^{\mathrm{s}}
\end{gathered}
$$

To arrive at eq 8 , it is sufficient to assume that the shear rate $\mathbf{S}=\partial \mathbf{u}_{\|} / \partial n$ has the same constant value, $\mathbf{S}_{0}$, up to the liquid-facing side of the interfacial layer. This is the case, for example, if numerically $\xi_{0} \ll 1$.

Equation 8 has a unique solution on $x \in \mathbb{R} \backslash 0$,

$$
\begin{gathered}
\rho^{\mathrm{s}}(x)=1 \mp\left(1-V_{0}\right) \frac{\mathrm{Ca}}{\xi_{0}} \exp \left(-\xi_{0}|x|\right), \quad \sigma(x)=\frac{\rho_{0}^{\mathrm{s}}}{\rho_{\mathrm{e}}^{\mathrm{s}}}-\rho^{\mathrm{s}}(x) \\
u_{x}(x)=1-\xi_{0}^{-2} \frac{\mathrm{d} \rho_{1}^{\mathrm{s}}}{\mathrm{d} x}=1-\left(1-V_{0}\right) \exp \left(-\xi_{0}|x|\right)
\end{gathered}
$$

provided that the surface phase flux is specified at the location of the nanothread, $x=0$

$$
\rho^{\mathrm{s}} v^{\mathrm{s}}=V_{0} \Leftrightarrow \frac{\mathrm{d} \rho_{1}^{\mathrm{s}}}{\mathrm{d} x}=\xi_{0}^{2}\left(1-V_{0}\right), \quad x=0
$$

(31) Hervet, H.; Leger, L. C. R. Phys. 2003, 4, 241-249. 
and the surface phase density tends to its equilibrium value, $\rho^{\mathrm{s}}=1$, away from the nanothread

$$
\rho_{1}^{\mathrm{s}} \rightarrow 0, \quad|x| \rightarrow \infty
$$

Here, the upper sign is for $x>0$, and parameter $V_{0}, 0 \leq V_{0} \leq 1$, is the normalized surface phase flux at $x=0$, which characterizes the strength of the particle-surface-phase-flux interactions. It is seen, from the asymptotic solution, that (a) substantial perturbations of the surface tension, sufficient to affect flow conditions at the substrate, exist over the distance $l_{0}=\lambda / \xi_{0}=\left(\lambda \tau \sigma_{0} / \mu\right)^{1 / 2}$ defined by the surface phase relaxation time, (b) the effect, generated by the particle, is simply proportional to the factor $1-V_{0}$ and vanishes at $V_{0}=1$, when the particle has no effect on the surface phase flow at all, and is maximal at $V_{0}=0$, when the particle blocks the flux in the interfacial layer.

In the current study, we elaborate on the simplified twodimensional analysis of the problem and consider the much more realistic three-dimensional situation of two-dimensional nanoparticles of an arbitrary shape, but with a simplified condition at the boundary $\Gamma$ of the obstacle, to obtain still informative results

$$
\left.\mathbf{v}^{\mathbf{s}} \cdot \mathbf{n}_{\mathbf{s}}\right|_{\Gamma}=0
$$

Here, $\mathbf{n}_{\mathrm{s}}$ is the external normal vector in the substrate plane to the boundary $\Gamma(x, y)=0$ (see Figure 1$)$.

The condition in eq 11, which is the main assumption in the model, implies that (a) the obstacle blocks the surface phase flux in the interfacial layer and (b) there is no contribution to the surface phase flux at the substrate from the flux generated over the area occupied by the obstacle. The latter is fulfilled if the surface phase flux generated over the top of the obstacle is negligibly small, because the size of the obstacle is small in comparison to the characteristic length scale of the interface formation, $l_{0}$; for the purpose of the current study, this is sufficient. Note here that the surface phase is different from the bulk liquid in the sense that it only exists at the substrate and, once being driven away from the boundary, becomes the bulk liquid, that is, it disappears.

It is possible, in general, that the flux at the obstacle boundary may not be zero and depend on the height and/or the shape of the particle. For example, it is expected that particles with the height smaller than the interfacial layer thickness would only partially disturb the flux of the surface phase. On the other hand, much larger particles, especially with a mushroom shape, would always create a stagnation zone with elevated pressure, by analogy to the common flows over rigid bodies in fluid mechanics, which can substantially or totally reduce the surface phase flux. Thus, the implication of our choice, condition 11, is that we will find in this general case an upper bound of the generated surface tensions with the help of the simplest and irreducible macroscopic theoretical model to obtain clear and informative results. From this point of view, condition 11 is a reasonable assumption to formulate a benchmark problem, which can be further generalized. On the other hand, such generalization is only meaningful if the surface phase flux is found quantitatively with the help of a microscopic consideration.

So, now, we consider the case of two-dimensional nanoparticles of an arbitrary shape with a smooth boundary $\Gamma_{k}(x, y)=0$ of the domain $\Omega_{k}$ occupied by the nanoparticle on the $(x, y)$ plane.
One can show that, in a similar way and in the same limit $\epsilon \rightarrow 0$, $\mathrm{Ca} / \epsilon=\xi_{0}^{2} \sim O(1)$, the problem in eqs $1-7$ and 11 can be reduced to an exterior Neumann boundary value problem for the modified Helmholtz equation on an unbounded domain, which is in the nondimensional form

$$
\begin{gathered}
\Delta \rho_{1}{ }^{\mathrm{s}}-\xi_{0}{ }^{2} \rho_{1}{ }^{\mathrm{s}}=0, \quad \mathbf{x} \in \mathbb{R}^{2} \backslash \underset{k}{\cup} \bar{\Omega}_{k} \\
\lim _{r \rightarrow \infty} \rho_{1}{ }^{\mathrm{s}}=0, \quad|\mathbf{x}|=r \\
\rho^{s}=1+\epsilon \rho_{1}{ }^{\mathrm{s}}
\end{gathered}
$$

with the boundary condition 11 , on $\Gamma=\cup_{k} \Gamma_{k}$ in the form

$$
\left.\frac{\partial \rho_{1}{ }^{\mathrm{s}}}{\partial n_{\mathrm{s}}}\right|_{\Gamma}=\xi_{0}{ }^{2} \mathbf{n}_{\mathbf{s}} \cdot \frac{\mathbf{S}_{0}}{S_{0}}, \quad \xi_{0}{ }^{2}=\frac{\mathrm{Ca}}{\epsilon}=\frac{\lambda^{2}}{l_{0}{ }^{2}}
$$

The tangential component of the velocity at the liquid-facing side of the interfacial layer can be expressed through $\rho_{1}^{\mathrm{s}}$ as

$$
\mathbf{u} \cdot(\mathbf{I}-\mathbf{n n})=\frac{\mathbf{S}_{0}}{S_{0}}-\xi_{0}^{-2} \nabla \rho_{1}{ }^{\mathrm{s}}
$$

so that even small perturbations of the surface density $(\sim \epsilon)$ will have strong effect on the flow conditions at the substrate.

\section{Numerical Method}

In general, a solution to eqs $12-13$ can be obtained numerically using a boundary integral method, ${ }^{32}$ which has superalgebraic or even exponential rate of convergence. This technique is especially advantageous if one has to deal with many particles distributed on the surface and is the basis for simulations in this study. In the method, the modified Helmholtz equation, eq 12, is represented in the integral form

$$
\begin{gathered}
\eta \rho_{1}{ }^{\mathrm{s}}(\mathbf{x})=\int_{\Gamma}\left\{\frac{\partial \rho_{1}^{\mathrm{s}}}{\partial n}(\mathbf{y}) \Phi_{\xi_{0}}(\mathbf{x}, \mathbf{y})-\frac{\partial \Phi_{\xi_{0}}}{\partial n}(\mathbf{x}, \mathbf{y}) \rho_{1}{ }^{\mathrm{s}}(\mathbf{y})\right\} \mathrm{d} s(\mathbf{y}), \\
\mathbf{y} \in \Gamma
\end{gathered}
$$

which involves values of $\rho_{1}^{\mathrm{s}}$ and its normal derivative only at the boundary

$$
\begin{gathered}
\eta=-1 \quad \mathbf{x} \in \mathbb{R}^{2} \backslash \cup_{k} \bar{\Omega}_{k} \\
\eta=-1 / 2 \quad \mathbf{x} \in \Gamma \\
\eta=0 \quad \mathbf{x} \in \cup_{k} \Omega_{k}
\end{gathered}
$$

$\Phi_{\xi 0}$ denotes the fundamental solution of 12

$$
\Phi_{\xi_{0}}=\frac{1}{2 \pi} K_{0}\left(\xi_{0}|\mathbf{x}-\mathbf{y}|\right), \quad \mathbf{x} \neq \mathbf{y}
$$

where $K_{0}$ is the modified Bessel function of the second kind of order zero.

Hence, if the Neumann boundary data, eq 13, is given, 15 is an integral equation for the unknown complementary data, which can be solved numerically. Once the complementary data have been found, eq 15 can be used again to calculate $\rho_{1}^{\mathrm{s}}$ anywhere in the domain $\mathbb{R}^{2} \backslash \cup_{k} \bar{\Omega}_{k}$. If $\Gamma_{k}$ are smooth, which is assumed, then eq 15 is solved numerically by a global approximation technique

(32) Langdon, S.; Graham, I. G. IMA Journal of Numerical Analvsis 2001, 21, 217-237. 
to achieve exponential convergence rate. ${ }^{32}$ That is, $\Gamma_{k}$ are parametrized by $2 \pi-$ periodic functions $\gamma_{k}(t): \mathbb{R} \rightarrow \Gamma_{k}, t \in[0,2 \pi]$, and $\rho_{1}^{\mathrm{s}}$ is discretized on each boundary $\Gamma_{k}$ as

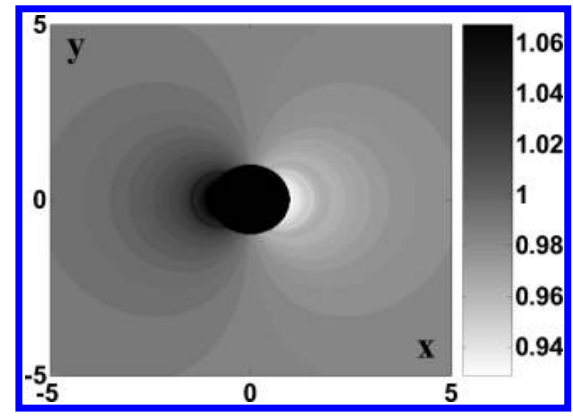

Figure 3. Distribution of the surface tension induced by a circular obstacle at $R_{0}=1, \theta_{0}=60^{\circ}, \epsilon=0.1$, and $\mathrm{Ca}=0.01$. The surface tension is normalized by its value in the far field $\sigma_{\infty}$ at $r \rightarrow \infty$ and the length scale is normalized by $\lambda$. The dark black area corresponds to the area occupied by the obstacle.

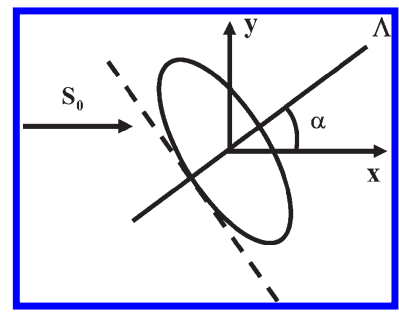

Figure 4. Definition sketch for the problem in the case of an asymmetric obstacle with arbitrary orientation with respect to the driving shear flow. The dashed line is to show the position of the line obstacle used in comparison.

$$
\rho_{1}^{\mathrm{s}}(t)=\sum_{j=0}^{2 n-1} a_{j} \phi_{j}(t), \quad t \in[0,2 \pi]
$$

$$
\begin{gathered}
\varphi_{k}(t)=\cos k t, \quad k=0, \ldots, n \\
\varphi_{k}(t)=\sin (k-n) t, \quad k=n+1, \ldots, 2 n-1
\end{gathered}
$$

Then, the problem is fully set to find, numerically, unknown coefficients $a_{j}$, which can be further used to calculate $\rho_{1}^{\mathrm{s}}$ everywhere in the domain (see more details in ref 32).

\section{Analysis of the Problem}

First, we consider just one circular obstacle of radius $R_{0}$ when the general analytical solution to eqs $12-13$ is available, which is, in a polar coordinate system with the origin at the center of the obstacle (Figure 1)

$$
\begin{gathered}
\rho^{\mathrm{s}}(r, \phi)=1+\epsilon A \cos (\phi) K_{1}\left(\xi_{0} r\right) \\
\sigma(r, \phi)=\frac{\rho_{0}{ }_{\mathrm{s}}}{\rho_{\mathrm{e}}^{\mathrm{s}}}-\rho^{\mathrm{s}} \\
A=-\xi_{0}\left(K_{0}\left(\xi_{0} R_{0}\right)+K_{1}\left(\xi_{0} R_{0}\right) / \xi_{0} R_{0}\right)^{-1}
\end{gathered}
$$

here, $K_{0}(z)$ and $K_{1}(z)$ are the modified Bessel functions of the second kind. If $\xi_{0} \ll 1$

$$
\begin{gathered}
\rho^{\mathrm{s}}(r, \phi) \simeq 1-\mathrm{Ca} R_{0} \frac{R_{0}}{r} \cos (\phi) \\
\sigma \simeq \frac{\rho_{0}{ }^{\mathrm{s}}}{\rho_{e}{ }^{\mathrm{s}}}-1+\mathrm{Ca} R_{0} \frac{R_{0}}{r} \cos (\phi)
\end{gathered}
$$

From this simple analytical result, one can see that, in general, the surface phase is compressed at the flow-facing side of the obstacle and is rarefied in the wake, as is the case in the two-

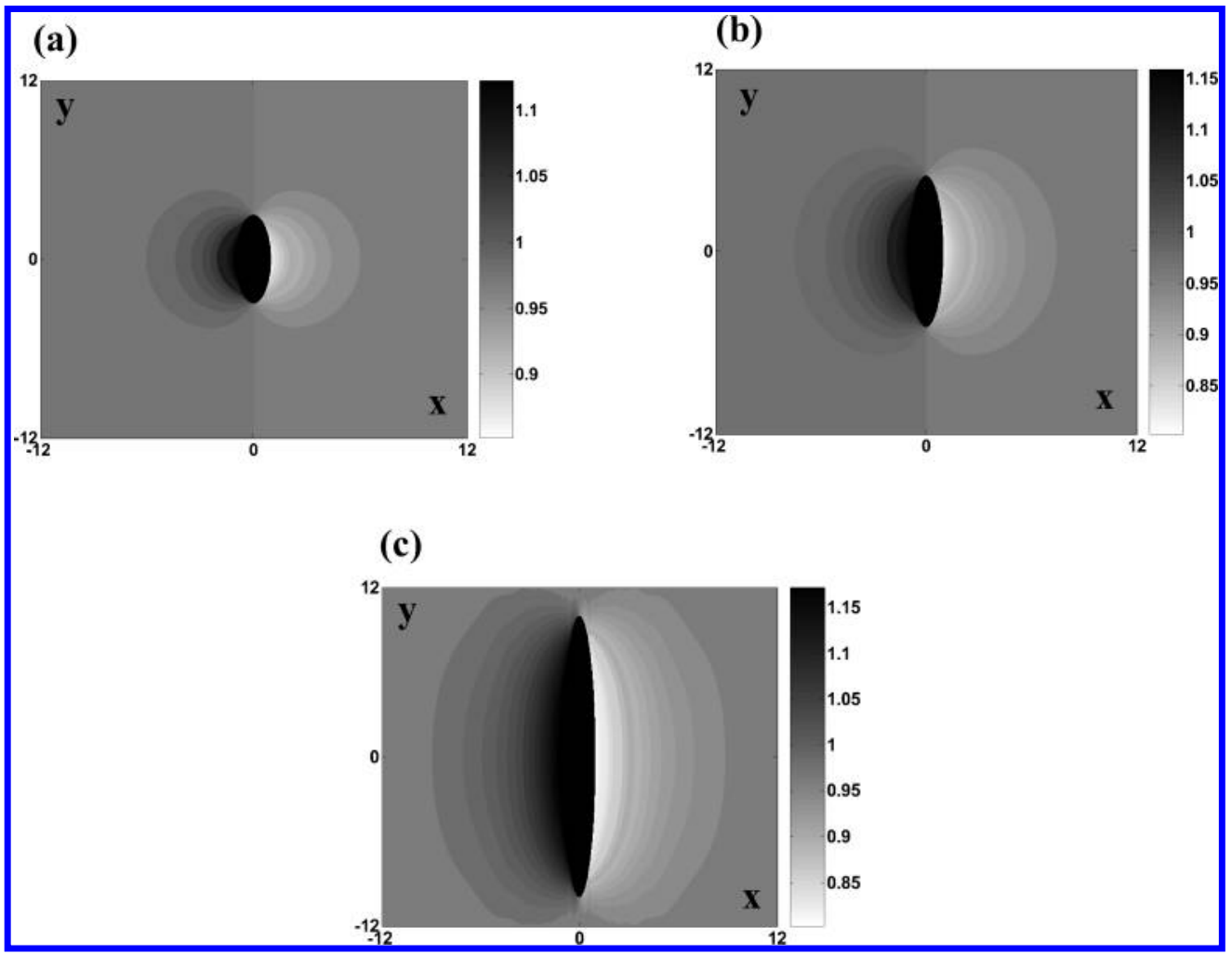

Figure 5. Distribution of the surface tension induced by a stretched obstacle at $\mathrm{Ca}=0.01, \epsilon=0.1, \theta_{0}=60^{\circ}$, and $\alpha=0$ and for the aspect ratios (a) $1: 3$, (b) $1: 5$, and (c) $1: 10$. The surface tension is normalized by its value in the far field $\sigma_{\infty}$ at $r \rightarrow \infty$ and the length scale is normalized by $\lambda$. The dark black area corresponds to the area occupied by the obstacle. 


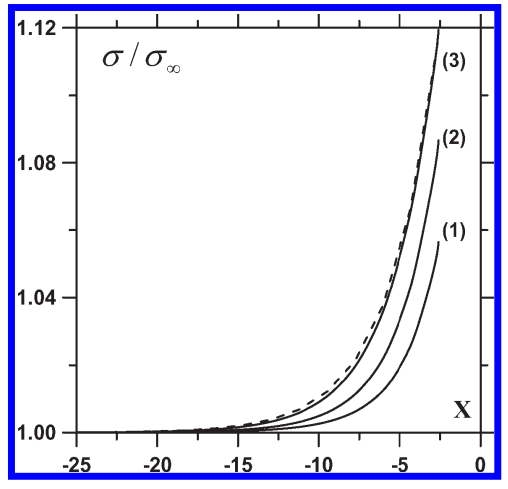

Figure 6. Distributions of the surface tension, solid lines, along the line $y=0$ induced by a stretched obstacle, as in Figure 5, at $\mathrm{Ca}=$ $0.01, \epsilon=0.1$, and $\theta_{0}=60^{\circ}$ and for the aspect ratios (1) $1: 3$, (2) $1: 5$, and (3) 1:10. The dashed line is the solution to eq 9 for the line obstacle located at $x=-1$ (dashed line in Figure 4). The surface tension is normalized by its value in the far field $\sigma_{\infty}$ at $r \rightarrow \infty$, and the length scale is normalized by $\lambda$.

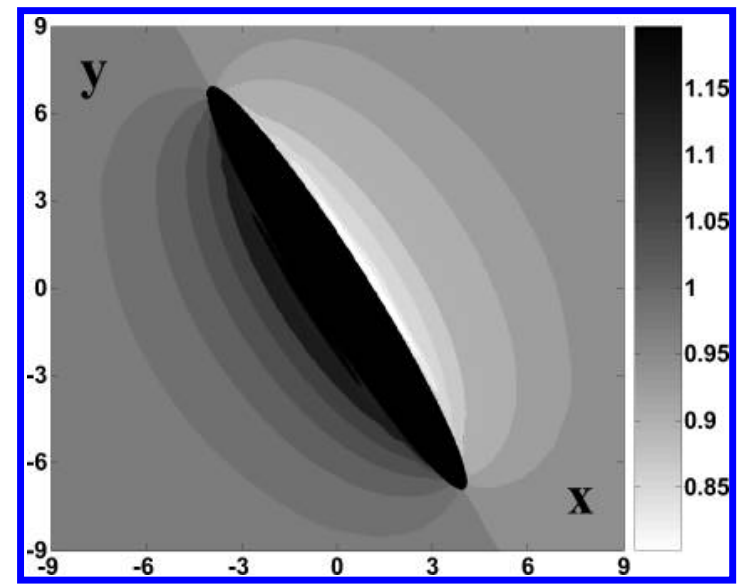

Figure 7. Distribution of the surface tension induced by a stretched obstacle with oblique orientation to the direction of the shear, $\alpha=30^{\circ}$, at $\mathrm{Ca}=0.01, \epsilon=0.1$, and $\theta_{0}=60^{\circ}$ and for the aspect ratio 1:8. The surface tension is normalized by its value in the far field $\sigma_{\infty}$ at $r \rightarrow \infty$, and the length scale is normalized by $\lambda$. The dark black area corresponds to the area occupied by the obstacle.

dimensional problem (9), and, for a circular-like object, (a) the topography-induced surface tension perturbations vanish with the size of the obstacle $R_{0}$, (b) the perturbation region is defined by the size of nanoparticles $R_{0}$ in contrast to the case of nanothreads (eq 9), where the characteristic distance is defined by the surface phase relaxation process (by the parameter $\xi_{0}$ ), (c) the strength of the effect is simply defined by the capillary number $\mathrm{Ca}$, that is, by the applied shear rate and the slip length, in contrast to the case of nanothreads (eq 9), where the effect is proportional to $\mathrm{Ca} / \xi_{0}$.

Consider for illustration a particular example of a viscous liquid from the range of PDMS fluids with $\mu=1 \mathrm{~Pa}$ s and $\sigma_{\mathrm{GL}}=$ $2 \times 10^{-2} \mathrm{~N} / \mathrm{m}$ at characteristic shear rate $S_{0} \sim 10^{4} \mathrm{~s}^{-1}$, when the liquid is still Newtonian. The characteristic slip length may be chosen in the range observed for this kind of fluids $\lambda \sim 100 \mathrm{~nm}^{31}$ The parameters $\tau$ and $\rho_{0} \mathrm{~s} / \rho_{\mathrm{e}}^{\mathrm{s}}$ (to calculate surface tension) are scaled, according to the estimates obtained from experiments on dynamic wetting, ${ }^{30}$ as $\tau=10^{-8}(\mu / 1 \mathrm{mPa} \mathrm{s}) \mathrm{s}, \rho_{0} \mathrm{~s} / \rho_{\mathrm{e}} \mathrm{s} \simeq[1+0.3$ $\left.\cos \left(\theta_{0}\right)\right]^{-1}$ for a liquid-solid combination with the static contact angle $\theta_{0}$. At $\theta_{0}=60^{\circ}$, parameters $\sigma_{0} \simeq 7 \times 10^{-2} \mathrm{~N} / \mathrm{m}, \epsilon \sim 0.1$, and $\mathrm{Ca} \sim 10^{-2}$, resulting in variations of the surface tension $\Delta \sigma / \sigma \sim$

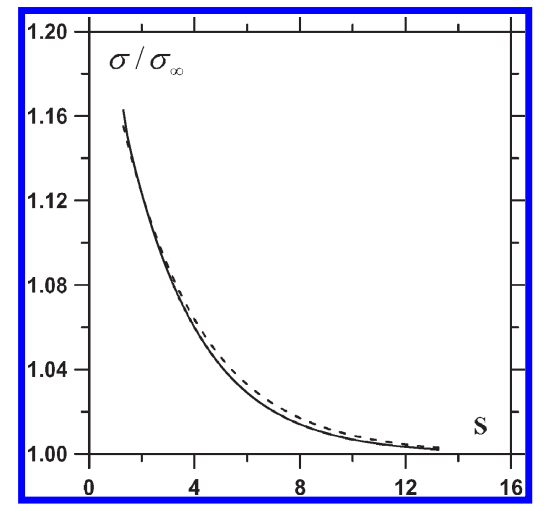

Figure 8. Distribution of the surface tension, solid line, induced by a stretched obstacle with oblique orientation to the direction of the shear, $\alpha=30^{\circ}$, calculated along the line $\Lambda$ shown in Figure 4 , at $\mathrm{Ca}$ $=0.01, \epsilon=0.1$, and $\theta_{0}=60^{\circ}$ and for the aspect ratio $1: 8$. The dashed line is the solution to eq 19 calculated along the same direction, $\Lambda$, and for the line obstacle as is shown by the dashed line in Figure 4. The surface tension is normalized by its value in the far field $\sigma_{\infty}$ at $r \rightarrow \infty$ and the distance $s$ is normalized by $\lambda$.

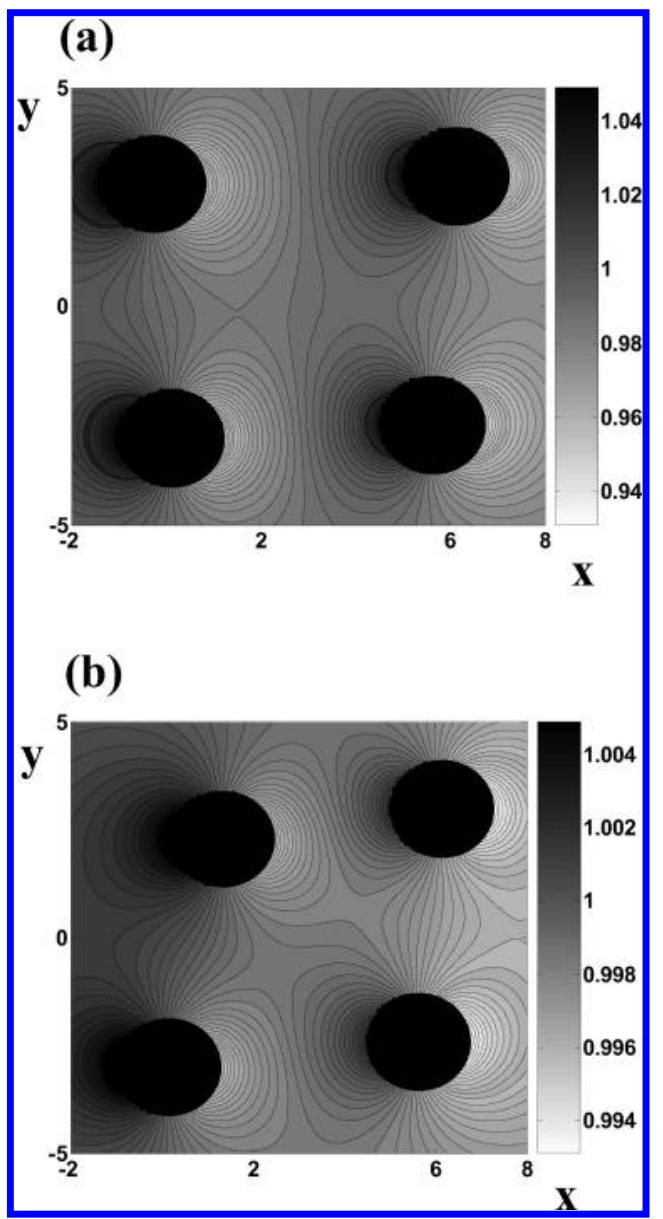

Figure 9. Typical distributions of the surface tension induced by a group of circular obstacles with $R_{0}=1$ at $\theta_{0}=60^{\circ}$ and (a) $\mathrm{Ca}=$ $0.01, \epsilon=0.1$, (b) $\mathrm{Ca}=0.001, \epsilon=0.1$. The surface tension is normalized by its value in the far field $\sigma_{\infty}$ at $r \rightarrow \infty$, and the length scale is normalized by $\lambda$. The dark black areas correspond to the areas occupied by the obstacles.

0.1 at $R_{0}=1$. Obviously, at higher viscosities, for larger obstacles $R_{0}>1$, and at static contact angles closer to $\theta_{0}=90^{\circ}$, the effect is much stronger, $\Delta \sigma / \sigma \sim 1$. The distribution of the generated surface tensions is illustrated in Figure 3. 


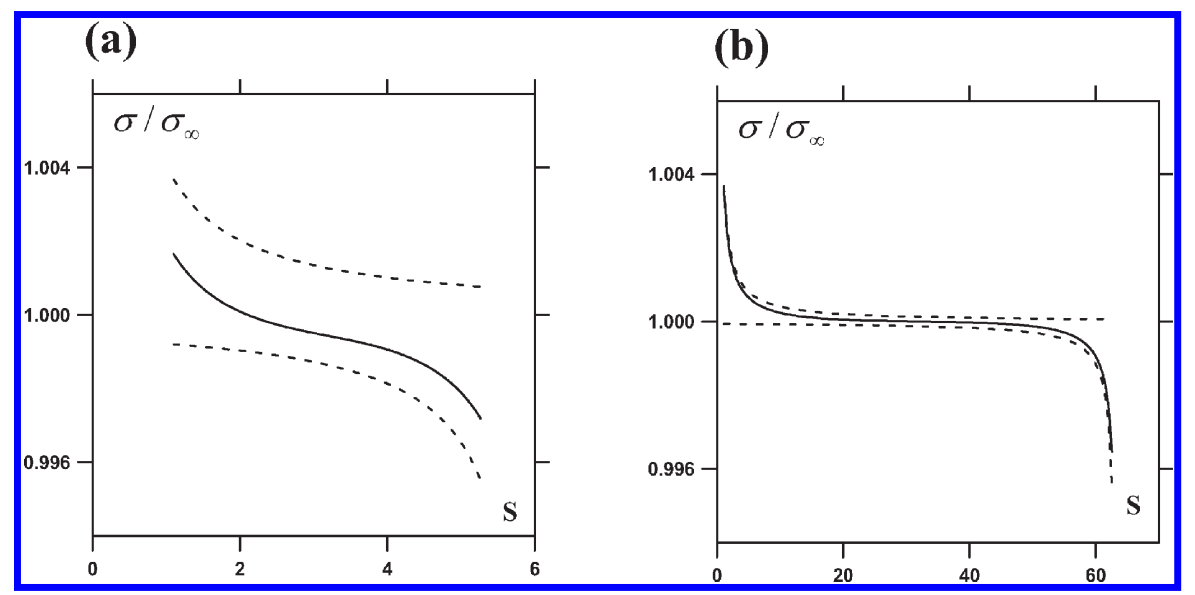

Figure 10. (a) Distribution of the surface tension (solid line) induced by the obstacles positioned identically to and with the same parameters as in Figure 9b, along the line connecting the centers of the two circular obstacles located in the opposite, upper left, and lower right corners. The dashed lines are the solutions calculated by means of eq 16 for each circular obstacle independently. The surface tension is normalized by its value in the far field $\sigma_{\infty}$ at $r \rightarrow \infty$, and the length $s$ is normalized by $\lambda$. (b) is identical to (a), but with all distances magnified ten times (keeping $\left.R_{0}=1\right)$.

Now, we will look at the effect of orientation and shape of the obstacle by considering single, but extended structures (see Figure 4 for illustration). One can expect that, while the aspect ratio of the largest obstacle size to the smallest one increases, the distribution of the surface tension will tend to the one described by the twodimensional result (eq 9). The obtained distributions are illustrated in Figure 5 for different aspect ratios of the obstacles. Qualitatively, one can observe that the effect becomes indeed stronger in general, while the obstacle is extended in the $y$-direction, as is expected from eq 9 at $\xi_{0} \ll 1$. Quantitatively, when the maximal obstacle size becomes larger than the characteristic length scale of the interface formation, $\xi_{0}^{-1}$, the distribution is effectively described by eq 9 . This can be clearly seen in Figure 6, if we plot the distributions of the surface tension, obtained for the same parameters and aspect ratios as in Figure 5, but just along the line $\Lambda$ (which is actually the line $y$ $=0$ in this case) as is shown in Figure 4.

If we now position the obstacle at an angle $\alpha$ to the direction of the driving shear flow, the effect on the surface tension distribution is basically reduced to the rotation of the whole picture in the same direction-deviations from the symmetric distribution are only seen in the far field (see Figure 7). Locally, when the maximal obstacle size is larger than the characteristic length scale of the interface formation, $\xi_{0}^{-1}$, the distribution, which is shown in Figure 8, is well-described by the two-dimensional result, (eq 9), though in this case being corrected to account for the oblique direction of the shear to the major obstacle axis

$$
\rho^{\mathrm{s}}(s)=1 \mp \cos \alpha \frac{\mathrm{Ca}}{\xi_{0}} \exp \left(-\xi_{0} s\right), \quad \sigma(s)=\frac{\rho_{0}{ }^{\mathrm{s}}}{\rho_{\mathrm{e}}^{\mathrm{s}}}-\rho^{\mathrm{s}}(s)
$$

Here, $\mathrm{s}$ is the distance from the line obstacle along the line $\Lambda$, as is shown in Figure 4, and the lower sign is for the flow-facing side of the obstacle.

Finally, we consider collective effects of clustered circular nano-obstacles. The typical results of simulations are presented in Figure 9 for different sets of parameters. The collective effects can be readily observed in the pictures of the surface tension distributions, while, at the same time, one can see that locally the phenomenon is largely similar to the one observed for a single circular obstacle. To quantify the collective effects, we have calculated the distribution of the surface tension along the line connecting the centers of the two obstacles located in the opposite corners in Figure $9 \mathrm{~b}$ and compared the result with the distributions calculated for each circular object by means of eq 16 independently (see Figure 10a). It is seen in Figure 10a that the distribution qualitatively resembles the results obtained by eq 16 , though the collective effects are strong enough and clearly visible. Now, if the system, shown in Figure 9b, is magnified as a whole ten times (but keeping $R_{0}=1$ ) to pull the obstacles further away, then the distribution, as is expected, can be well-described by eq 16 calculated for each object independently (see Figure 10b). So, one can say that the collective effects are important when the obstacles are separated by the distance, which is comparable with the characteristic size of the perturbation region. This region is defined, in general, by the parameter $\xi_{0}{ }^{-1}$, but note that, for symmetric circular-like objects, the characteristic size is defined by the obstacle size only.

\section{Conclusions}

In conclusion, it has been shown that nanoscale topography of the substrate, with the height of irregularities on the length scale of the interfacial layer, may be a significant factor in the generation of noticeable dynamic surface tension stresses, which cannot be ignored in simulations of flows at nanoscale. The amount of the effect and its spatial distribution depend on the surface phase and solid substrate parameters, such as slip length and relaxation time, the shape and the size of the obstacles and last, but not least, on the strength of the obstacle-surface-phase-flux interaction. The last dependency is currently under detailed investigation by means of molecular dynamics simulations and will be the subject of further publications. In addition to the above, the greatest effect is found in the case of asymmetric extended structures or nanothreads. The collective effect of many obstacles will amplify the generated stresses in general, although it is too early to make any definite conclusions or implications concerning very rough surfaces.

The phenomenon has important repercussions, since the flowinduced surface tensions directly contribute to the boundary conditions for the motion of the bulk fluid and have strong effects on the hydrodynamic velocity at the substrate, even if the surface phase density is only slightly perturbed $\delta \rho^{\mathrm{s}} \sim \epsilon$, as is seen from eq 14. The latter implies that the analysis of generated surface tensions, presented here, is also crucial for interpretation of slip length measurements.

Acknowledgment. The author is very grateful to S. Langdon for his help with numerical simulations. 